The Be Phenomenon in Early-Type Stars, IAU Colloguium 175

ASP Conference Series, Vol. 214, 2000

M. A. Smith, H. F. Henrichs, and J. Fabregat, eds.

\title{
He I Line Profiles Obtained From a Be Wind Model
}

\author{
R. Rohrmann ${ }^{1}$ \\ Observatorio Astronómico, Laprida 854, 5000 Córdoba, Argentina
}

\begin{abstract}
We undertook a non-LTE radiative transfer calculation to find out the possible conditions under which observed $\mathrm{H}$ and $\mathrm{He} \mathrm{I}$ line profiles arise from some Be stars.
\end{abstract}

\section{Introduction}

Emission from He I lines has been found in several Be stars, for example $\lambda \lambda 5876$, 10830,20581 in $\gamma$ Cas (Lowe et al. 1985, Chalabaev and Maillard 1985), $\lambda 5876$ in $\kappa \mathrm{CMa}$, o Pup, HR 4830 and HR 2855 (Dachs et al. 1992), $\lambda \lambda 6678,5876,4922$, 4471 in $\lambda$ Eri (Smith et al. 1994), and $\lambda 6678$ in $\mu$ Cen (Rivinius et al. 1998). Some of these stars undergo erratic episodes, or outbursts, of Balmer and $\mathrm{He} \mathrm{I}$ line emission, which reveal that complex processes occur in their atmospheres. A simultaneous calculation of profiles for several lines can give information on the structure of the outer layers of these stars. Moreover, a more realistic restriction on the atmospheric model could be obtained if the prediction of several lines is required.

Our main objective is to investigate if the observed behavior of the He I line emission can arise from an expanding atmosphere, with a given density and temperature distribution. We present results on the formation of some $\mathrm{He} \mathrm{I}$ and $\mathrm{H}$ lines, mainly $\lambda \lambda 6678,5876$, and $\mathrm{H} \alpha$.

\section{The model}

We have written a code which solves the continuum and line radiative transfer equations consistently with the statistical equilibrium equations, for a pure hydrogen-helium plasma, applying the equivalent-two-level-atom method. The He I atom includes 11 NLTE singlet and triplet states. The H I and He II atoms consist of six NLTE levels each. All relevant atomic processes were taken into account.

We assumed a spherically symmetric steady-state wind. Although this model is not the best representation of the atmosphere of rotating stars, it allows a rigorous treatment of the line transfer problem by using the comoving frame method (Mihalas and Kunasz 1978). We can expect to derive average information about the line formation from this analysis. The photosphere is represented

${ }^{1}$ Fellow of the CONICET, Argentina 
with a density and temperature distribution taken from Kurucz (1979), as they are considered as parameters in the wind.

\section{Application}

We have studied a part of the model parameter space corresponding mainly to the formation of double-peaked profiles, which were found, for example, in the red lines $\lambda \lambda 5876$ and 6678 from observations of $\lambda$ Eri (Smith et al. 1994) and also in $\mathrm{H} \alpha$ and $\lambda 6678$ during a quiescence stage of $\mu$ Cen (Rivinius et al. 1998). We have chosen a reference model with $T_{\text {eff }}=20000 \mathrm{~K}$ and $\log g=3.5$ to show the behavior of the calculated profiles. The most representative results are presented here.

Two velocity laws were adopted

$$
\begin{gathered}
v(r)=v_{\infty} /\left[1+\left(a r_{\mathrm{ph}} / r\right)^{\alpha}\right] \quad(\text { Law } \alpha), \\
v(r)=v_{\infty}\left(1-b r_{\mathrm{ph}} / r\right)^{\beta} \quad(\text { Law } \beta) .
\end{gathered}
$$

They are computed according to the mass-loss rate $\dot{M}$, the terminal velocity $v_{\infty}$, the density at the photospheric radius $r_{p h}$ and the condition $\tau_{2800}=2 / 3$. The velocity law $\alpha$ usually reaches the asymptotic velocity close to the photosphere; on the contrary, the velocity law $\beta$ has a very low gradient.

\section{Results}

Table 1 lists some models calculated. We call $r_{v}$ the radius at the maximum gradient of the velocity. All models have $v_{\infty}=300 \mathrm{~km} / \mathrm{s}$.

Table 1. Properties of the models.

\begin{tabular}{ccccc} 
Model & $T_{\min }[\mathrm{K}]$ & $\dot{M}\left[\mathrm{M}_{\odot} / \mathrm{yr}\right]$ & Velocity law & $r_{v} / r_{\mathrm{ph}}$ \\
\hline $\mathrm{a}$ & 13700 & $2 \times 10^{-10}$ & $\beta$ & 1.88 \\
$\mathrm{~b}$ & 14300 & $1 \times 10^{-10}$ & $\beta$ & 1.97 \\
$\mathrm{c}$ & 13900 & $1 \times 10^{-10}$ & $\alpha$ & 1.03 \\
$\mathrm{~d}$ & 14300 & $1 \times 10^{-10}$ & $\alpha$ & 1.03
\end{tabular}

Typical double-peaked profiles appear for models with low $\dot{M}$ and wind temperature rising from a minimum value $T_{\min }$ against the photosphere up to a maximum value of $30000 \mathrm{~K}$ in the outer regions (Figure 1).

The emission in the wings and the central absorption depend both on the temperature and the velocity gradients at the base of the wind; $\lambda 6678$ in particular is very sensitive to these gradients. In these models photospheric absorption wings are often visible in some lines such as $\mathrm{H} \alpha, \lambda \lambda 10830$ and 5876. All the subordinate lines are formed very near to the photosphere where the resonance lines are still opaque and the electron density is between $10^{10}$ and $10^{13} \mathrm{~cm}^{-3}$ 



Figure 1. Emergent flux profiles from different models.

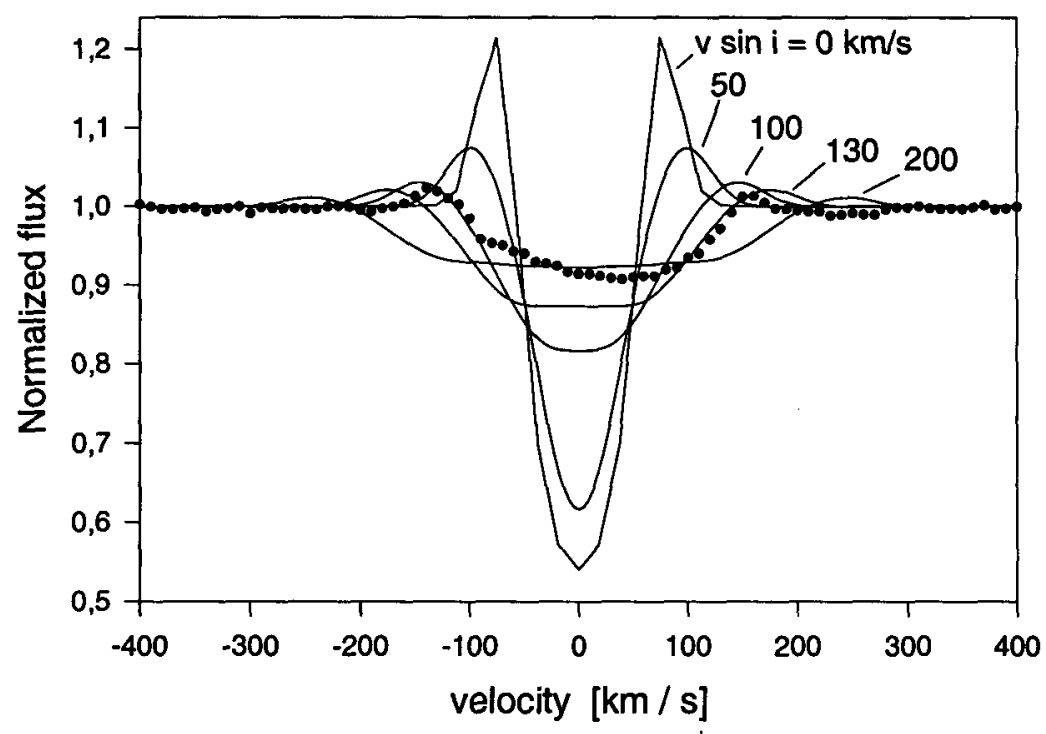

Figure 2. The He I $\lambda 6678$ from model d for different projected rotational velocities, and the observed profile (filled symbols) during a relative quiescence phase of $\mu$ Cen (Rivinius et al.). 
(see Table 2). For high values of $\dot{M}$ the formation regions occur more outwards and P Cygni profiles are obtained for some analyzed lines.

Figure 2 presents the $\lambda 6678$ profile from one of the models including Doppler effect for various projected rotational velocities $v \sin i$. In an exploratory way these broadening profiles are compared with one observed in $\mu$ Cen during a relative quiescence phase (Rivinius et al. 1998). The separation between the double emission peaks suggests a projected rotational velocity of $100-130 \mathrm{~km}$ $\mathrm{s}^{-1}$, in agreement with the value derived by Brown and Verschueren (1997) from high-S/N echelle spectroscopy.

Table 2. Formation regions of some lines obtained from model b.

\begin{tabular}{rcrcrcrc} 
Line & $r / r_{\mathrm{ph}}$ & Line & $r / r_{\mathrm{ph}}$ & Line & $r / r_{\mathrm{ph}}$ & Line & $r / r_{\mathrm{ph}}$ \\
\hline$\lambda 537$ & $1.15-1.60$ & $\lambda 6678$ & $1.02-1.11$ & $\mathrm{~L} \alpha$ & $1.07-1.30$ & $\mathrm{H} \alpha$ & $1.03-1.11$ \\
$\lambda 584$ & $1.11-1.42$ & $\lambda 7065$ & $1.02-1.08$ & $\mathrm{~L} \beta$ & $1.03-1.16$ & $\mathrm{H} \beta$ & $1.03-1.11$ \\
$\lambda 3389$ & $1.01-1.05$ & $\lambda 7281$ & $1.01-1.05$ & $\mathrm{~L} \gamma$ & $1.04-1.15$ & $\mathrm{H} \gamma$ & $1.01-1.05$ \\
$\lambda 5016$ & $1.01-1.03$ & $\lambda 10830$ & $1.03-1.11$ & $\mathrm{~L} \delta$ & $1.03-1.15$ & $\mathrm{H} \delta$ & $1.01-1.03$ \\
$\lambda 5876$ & $1.03-1.11$ & $\lambda 20581$ & $1.01-1.08$ & $\mathrm{~L} \epsilon$ & $1.01-1.08$ & &
\end{tabular}

\section{Discussion}

Our main result is that double-peaked profiles such as observed in $\lambda \lambda 5876$ and 6678 for $\lambda$ Eri and $\mu$ Cen, could arise from the base of a low density wind with a temperature increasing from the photospheric value. The calculated profiles are symmetric. Nonhomogeneous density and velocity distributions in the line formation regions could be the cause of the changes in the violet/red emission ratio observed in some He I lines.

Acknowledgments. I wish to thank G. Carranza for his thorough reading of the manuscript and valuable suggestions.

\section{References}

Brown, A.G.A., Verschueren, W. 1997, A\&A 319, 811

Chalabaev, A.A., Maillard, J.P. 1985, ApJ 294, 640

Dachs, J., Hummel, W., Hanuschik, R.W. 1992, A\&AS 95, 437

Kurucz, R. 1979, ApJS 40, 1

Lowe, R.P., Moorhead, J.M., Wehlau, W.H., Barker, P.K., Marlborough, J.M. 1985, ApJ 290, 325

Mihalas, D., Kunasz, P.B. 1978, ApJ 219, 635

Rivinius, Th., Baade, D., Stefl, S., Stahl, O., Wolf, B., Kaufer, A. 1998, A\&A 333,125

Smith, M.A., Hubeny, I., Lanz, T., Meylan, T. 1994, ApJ 432, 392 